\title{
Competencias científicas en estudiantes de grado 4to. y 5to. de primaria
}

Scientific competencies in 4to. and 5to. grade students

\author{
Gisele Carolina Pabón Guevara
}

caritopg625@gmail.com

Código ORCID: 0000-0001-8387-6881

Universidad Metropolitana Educación, Ciencia y Tecnología

(Umecit), Panamá

Recibido abril 2020 | Arbitrado mayo 2020 | Publicado junio 2020

\section{Resumen}

Palabras clave:

Científicas;

competencias

cognitivas;

competencias

procedimentales;

competencias

actitudinales

La presente investigación tuvo como objetivo describir las competencias científicas de los estudiantes de los grados cuarto y quinto de la institución educativa Gabriel García Márquez del municipio de Soacha, departamento de Cundinamarca. Se considera una investigación de tipo descriptiva, con un diseño de campo, transeccional contemporáneo univariable. La población la conforman 102 estudiantes: 52 de cuarto y 50 de quinto de la institución estudiada. Para la recolección de los datos se empleó como instrumento una evaluación de las competencias científicas basada en las pruebas "Saber", contentiva de 40 ítems de alternativas de respuestas múltiples. La validez se realizó mediante la técnica de validación, por tres expertos en ciencias y la confiabilidad se calculó mediante el Alfa de Cronbach, obteniéndose un valor de 0,75 lo que indica que el instrumento es altamente confiable. Los resultados indican que los estudiantes sólo poseen el $75 \%$ de las competencias científicas, requeridas por el nivel educativo en el cual se encuentran.

\section{Abstract}

Keywords:

Scientists; cognitive competencies;

procedural competencies; attitude competencies
This research aims to describe the scientific competencies of students in the fourth and fifth grades of the Gabriel García Márquez educational institution in the municipality of Soacha, Cundinamarca department. It is considered a descriptive type research, with a field design, unique contemporary transactional. The population consists of 102 students: 52 of fourth and 50 of fifth of the institution studied. For the collection of data, an assessment of scientific competences based on the evidence "Saber", contentive of 40 items of multi-response alternatives, was used as an instrument. The validity was done using the validation technique, by three science experts and reliability was calculated using the Cronbach Alpha, obtaining a value of 0.75 indicating that the instrument is highly reliable. The results indicate that students have only $75 \%$ of the scientific skills required by the level of education in which they are located 


\section{INTRODUCCIÓN}

Son muchas las formas en que se puede concebir el término ciencia; sin embargo, una manera muy general de conceptualizarla es como: un conjunto organizado de contenidos, métodos y técnicas para la adquisición y sistematización de conocimientos, sobre la estructura de un conjunto de hechos, fenómenos y objetivos accesibles a varios observadores. También puede agregarse que la ciencia es producto de la investigación de los fenómenos naturales y sus causas.

Independientemente del concepto que se maneje, algo es claro: la ciencia avanza solamente a través de la investigación científica, pues ella ha permitido al ser humano hacer una reconstrucción conceptual de la realidad, que es cada vez más amplia, profunda y exacta. Es así como el ser humano domina y moldea la naturaleza, sometiéndola a sus propias necesidades; reconstruye la sociedad y es, a su vez, reconstruido por ella; tratando luego de remodelar ese ambiente artificial para adaptarlo a sus propias necesidades.

Posiblemente, la investigación científica resulte ajena a la mayoría de las personas, pero lo cierto es que gran parte del conocimiento que se genera en ella tiene aplicaciones muy familiares, de uso muy común y cotidiano. Es decir, el hombre ha cambiado su diario vivir gracias a los adelantos tecnológicos que se han producido; primero, por el conocimiento de fenómenos que han conducido al desarrollo de la tecnología y con ello el avance de la ciencia.

Es así como la tecnología ha aportado instrumentos usados en diferentes ciencias: el telescopio, el microscopio, el computador, localizador, entre muchos otros que facilitan y mejoran el proceso de observación propio de la ciencia. De allí que puede decirse que entre la ciencia y la tecnología existe una relación de mutuo apoyo; así como la existente entre la educación y la investigación.

Esa estrecha y obligatoria relación entre la educación y los adelantos de la ciencia, llevan a dirigir la mirada hacia la educación que se ofrece en Colombia en el área de Ciencias Naturales, ya que la formación de los nuevos investigadores surge primero desde las escuelas; es decir, es en los grados de básica donde los niños y jóvenes comienzan a observar el maravilloso mundo del ambiente, la física, la química y todos los fenómenos que le rodean.

Según la visión y los intereses de los organismos y entidades que las promulgan, las competencias científicas, al igual que las competencias en general, tienen diferentes definiciones. Por ejemplo, la competencia científica, según el Programa Internacional de Evaluación a Estudiantes, Ministerio de Educación. (2009), es el uso que hace el individuo de los saberes científicos, para explicar los fenómenos y adquirir nuevos conocimientos. Igualmente, para el Banco Mundial (2014) es la posibilidad de participación en la economía mundial, esta definición deja de lado la parte pedagógica y humana y se centra en las capacidades del saber y el hacer, enfocadas a la productividad.

Como se evidencia, el estudio de las competencias es amplio hacia todas las áreas del conocimiento; de tal forma y con tanto interés, que incentiva a los países latinoamericanos a trabajar en el desarrollo de competencias orientadas a formar 
individuos integrales, con capacidades múltiples para resolver los problemas del contexto socio-económico de la región.

En Colombia, en 1998, el Ministerio de Educación Nacional (MEN) publica los Lineamientos Curriculares, los cuales establecen el horizonte epistemológico y pedagógico de la educación basada en competencias. Al respecto, Vallejo (2014) refiere que en el 2004 el MEN publica la primera edición de los Estándares Básicos de Competencias y en el 2006 la segunda edición, introduciendo así formalmente el término de competencia científica en el ámbito escolar del país.

En función de su contenido integral, el objetivo de las competencias científicas no es solamente formar científicos $o$ expertos en un área específica, sino que involucra la formación de ciudadanos con conocimientos, habilidades y valores, que comprendan y puedan responder responsable y conscientemente a los avances científicos y problemáticas ambientales que presenta el mundo actual.

Para lograr que los estudiantes sean competentes en ciencias se deben formar desde las etapas iniciales; es decir, desde la primaria, ya que, como menciona Di Mauro et al., (2015), es importante formar científicamente al individuo desde la básica ya que de esto depende el éxito o el fracaso futuro en las ciencias en general, toda vez que es en ese nivel donde se sientan las bases para los futuros científicos.

En Colombia, con la publicación de los Lineamientos y los Estándares en Ciencias, se da un horizonte común a la educación científica en el país con un enfoque en competencias, ya que aunque cada institución es autónoma en su currículo, estos documentos sirven de guía para dirigir la enseñanza de las ciencias naturales y preparar a los estudiantes para las pruebas nacionales "Saber".

En este sentido, los resultados de las pruebas "Saber" 3. $\stackrel{\circ}{5 .} .^{\circ}$ y $9 .^{\circ}$ son de vital importancia para esta investigación ya que el propósito principal de las mismas es contribuir al mejoramiento de la calidad de la educación colombiana, mediante la realización de evaluaciones aplicadas periódicamente para monitorear el desarrollo de las competencias básicas en los estudiantes de educación básica. Las pruebas "Saber" son una reconocida evaluación que realiza permanentemente, en Colombia, el Instituto Colombiano para el Fomento de la Educación Superior (Icfes), por solicitud del Ministerio de Educación Nacional (MEN).

De esta manera, las pruebas "Saber" son un referente nacional para conocer las condiciones de la educación en las diferentes regiones, instituciones y niveles educativos. En lo que concierne a las ciencias naturales las pruebas "Saber" evalúan tres competencias las cuales son: uso compresivo del conocimiento científico, explicación de los fenómenos e indagación sobre temas del área de ciencias.

Estas pruebas posibilitan conocer cuando los estudiantes tienen dificultades en competencias de lectura, matemáticas y ciencias, lo que orienta al Ministerio de Educación Nacional, para tomar medidas mediante la creación de programas como es el caso de "Todos a Aprender" (PTA), el cual está focalizado hacia instituciones educativas con bajos resultados en las pruebas, y por medio del cual se da a los docentes estrategias en Lenguaje $y$ 
Matemáticas, para aumentar, de esta manera, las competencias de los estudiantes en estas áreas, dejando de lado las competencias científicas.

En la institución educativa Gabriel García Márquez, ubicada en el municipio de Soacha, se implementa desde hace cinco años el Programa Todos a Aprender (PTA), que fortalece las competencias de lectoescritura y matemáticas de los estudiantes de primaria; sin embargo, todavía se observan grandes dificultades en los estudiantes para comprender y resolver situaciones relacionadas con la ciencia, lo que se evidencia en los resultados obtenidos en las pruebas "Saber" de ciencias para el grado quinto que se realizaron en los años 2014 y 2016, donde se obtuvo resultados del $67 \%$ y el $65 \%$ respectivamente, que se refieren a un nivel de desempeño mínimo, según lo publicado en esos años por el Instituto Colombiano para el Fomento de la Educación Superior (Icfes).

Es así como a los estudiantes de grado cuarto de la unidad educativa Gabriel García Márquez, aunque les causa curiosidad algunos fenómenos que ocurren a su alrededor, ven las ciencias como algo alejado de su realidad, se les dificulta plantear hipótesis, observar con visión crítica, analizar, buscar explicaciones o diseñar experimentos, por lo cual siempre esperan que sea el docente quien dé respuesta a sus interrogantes.

La situación antes mencionada hace muy difícil el trabajo cooperativo ya que algunos estudiantes no respetan las ideas de los demás, no comparten o no comunican sus inquietudes y expectativas en forma abierta y clara, esto es muy evidente durante las clases de ciencias, así como también en las evaluaciones y talleres cuando el docente les pide que relacionen lo aprendido para realizar planteamientos o resolver situaciones de la vida cotidiana.

Con base a lo descrito, el presente artículo tiene como objetivo central dar respuesta a la siguiente pregunta de investigación: ¿Cuáles son las competencias científicas de los estudiantes de los grados cuarto y quinto de la I E Gabriel García Márquez, sede $\mathrm{B}$, jornada tarde?

\section{Fundamentación teórica}

La educación científica se enfoca en el estudio y preparación de individuos en ciencia. Es una dimensión fundamental de la sociedad, ya que a través de ella los seres humanos han tratado de explicar el mundo, las relaciones y fenómenos que en él se dan $\mathrm{y}$, tal como menciona Hernández (2005), las ciencias son reconocidas como bienes culturales preciosos, por su impacto en la vida y en la producción, lo cual hace importante que todos los ciudadanos accedan a ella.

Tradicionalmente la ciencia se ha relacionado únicamente con los científicos, a pesar de que sus avances, descubrimientos y teorías inciden directamente en la vida de todos los seres humanos. Hernández, (2005) considera que no sólo los científicos, sino todos los ciudadanos establecen una relación con la ciencia, puesto que el mundo cambia por la ciencia, las tecnologías de la comunicación y la información.

El avance científico y social al que se enfrenta el mundo en la actualidad, exige que no sólo los científicos se interesen por las ciencias, sino que los ciudadanos en general comprendan y participen en temas científicos, ya que según consideración de Hernández (2005), la enseñanza básica de las ciencias es esencial para el hombre de 
hoy que desea entender su entorno y participar en las decisiones sociales.

Es así como la escuela por ser ente responsable de la educación formal está llamada a formar ciudadanos que comprendan su entorno, que participen y que tengan conocimientos en ciencias, para que respondan a las necesidades científicas del mundo de hoy, dado que, como menciona la Unesco (2009), en la actualidad la educación científica necesita un gran número de individuos con comprensión científica para el trabajo y participación ciudadana.

Para formar individuos científicamente que respondan a las demandas de la sociedad actual, la educación científica debe tener claros sus objetivos, los cuales son según Pozo y Gómez (2009): despertar en los estudiantes interés y favorecer las relaciones entre las formas de conocimiento cotidiano y científico. Mientras que para la Unesco (2009) "el objetivo fundamental de la educación científica es que el estudiante obtenga una perspectiva coherente, que entienda, aprecie, pueda relacionarse con el mundo que lo rodea y le sea útil para manejarse en su vida cotidiana" (p.41).

Del mismo modo, la Unesco (2006, p.23, c.p. Unesco 2009) menciona que:

El objetivo primordial de la educación científica es formar a los alumnos para que sepan desenvolverse en un mundo impregnado por los avances científicos y tecnológicos, para que sean capaces de adoptar actitudes responsables, tomar decisiones fundamentadas $\mathrm{y}$ resolver los problemas cotidianos desde una postura de respeto por los demás, por el entorno $y$ por las futuras generaciones que deberán vivir en el mismo.

Estos objetivos se podrían lograr y la educación científica sería eficaz si, como mencionan Pozo y Gómez (2009), los métodos de enseñanza, contenidos y metas, toman en cuenta el saber disciplinar, las características de los estudiantes a los que va dirigido $\mathrm{y}$ las demandas sociales $\mathrm{y}$ educativas en las que esa enseñanza tiene lugar, además de potenciar en los jóvenes formas de pensamiento complejas (pensamiento formal), en vez de proporcionar muchos conocimientos nuevos específicos.

El desarrollo del pensamiento formal, plasmado en los postulados de Piaget, según explican Pozo y Gómez (2009), se da cuando el individuo imagina otras posibilidades más allá de lo inmediato y real y trabaja con ellas como modelos hipotéticos representados en un lenguaje formal, lo que sin lugar a dudas conduce a que tenga pensamientos complejos, así como ideas variadas sobre el mismo problema o situación a estudiar.

De tal manera que Pozo y Gómez (2009) explican que el pensamiento formal de Piaget, se puede considerar como la descripción psicológica del pensamiento científico, que consiste en un análisis de procesos $y$ estructuras necesario para enfrentarse a la realidad con la mente de un científico.

En Colombia las ciencias se encuentran dentro del currículo oficial y como indica Hernández (2005) son reconocidas en la escuela como prácticas sociales, lo que se evidencia en los estándares que tienen en cuenta el componente de ciencia y tecnología, y que son usados por las 
instituciones educativas como base para crear el currículo de las asignaturas en ciencias de cada grado.

Asimismo, en la Ley 115 de 1994, se precisan los fines de la educación en ciencias naturales en Colombia. En ese documento, específicamente, en el artículo $5^{\text {a }}$ numerales $5,7,9,13$, se señalan los siguientes fines: Quinto; adquisición y generación de conocimientos científicos y técnicos más avanzados, mediante la apropiación de hábitos intelectuales adecuados para el desarrollo del saber. Séptimo; acceso al conocimiento, la ciencia, la técnica y el fomento de la investigación. Noveno; desarrollo de la capacidad crítica, reflexiva y analítica que fortalezcan el avance científico y tecnológico nacional orientado con prioridad al mejoramiento cultural y de la calidad de la vida. Decimotercero; promoción en la persona de la capacidad, investigar, adoptar la tecnología que se requiere en los procesos de desarrollo del país.

En Colombia, el Ministerio de Educación (1998) planteó los Lineamientos Curriculares en Ciencias como orientaciones conceptuales, pedagógicas y didácticas, para el diseño curricular, desde preescolar hasta la educación media. Del mismo modo, Vallejo (2014) expresa que los Lineamientos Curriculares son el documento oficial que establece el horizonte epistemológico y pedagógico de la educación científica escolar.

Bajo esa óptica, para el MEN (1998) el área de ciencias naturales y educación ambiental debe ofrecer a los estudiantes colombianos la posibilidad de conocer los procesos físicos, químicos y biológicos; además de su relación con los procesos culturales, en especial aquellos que puedan afectar de alguna manera el medio ambiente.

A pesar de los objetivos propuestos por el ente rector, la existencia de los estándares y lineamientos en ciencias y de que, como indica Rojas (2008), en el gobierno del 2002 al 2008 se dio la llamada revolución educativa en la cual se retomaba la formación en investigación, la educación científica en el país sigue siendo aún precaria, lo cual puede ser consecuencia como mencionan Pozo y Gómez (2009) de que el currículo en Ciencias ha tenido muy pocas modificaciones, mientras que la sociedad a la que va dirigido y las demandas de formación de los estudiantes han cambiado mucho.

El notable atraso en ciencias del país, muestra una escasa capacidad para el vínculo entre Educación-Sociedad-Estado, lo cual se refleja en los bajos resultados en las pruebas de ciencia, la poca motivación de los estudiantes y en indicadores como los mostrados por Arana (2005, p.297) “donde sólo el $1 \%$ de los científicos del mundo son latinoamericanos, y de estos sólo el $0.01 \%$ son colombianos, donde un alto porcentaje emigra a los países desarrollados, en detrimento de la ciencia y del país en general".

Para solventar los problemas en la educación científica en Colombia, como menciona Rojas (2008), se estimuló a través de políticas públicas la creación del Instituto Colombiano para el Fomento de la Educación Superior (Icfes) y el Instituto Colombiano para el Desarrollo de la Ciencia y la Tecnología (Colciencias). Entidades orientadas hacia el desarrollo de las ciencias en el país, que han creado programas extracurriculares para involucrar al sector educativo en el productivo, e incentivar al 
gobierno en la formación en masa de investigadores.

Entre los programas que apoyan la educación científica en las instituciones se encuentran, según explica Rojas (2008): el programa Pequeños Científicos, implementado en Colombia desde 1998 por la Universidad de los Andes, Maloka, el liceo Luis Pasteur y el Programa Ondas, que fue adelantado en 2005 por Colciencias con el apoyo de diversas instituciones, con el propósito de responder al atraso científico y tecnológico del sistema educativo colombiano.

Estos programas, aunque muestran resultados positivos, no llegan a todas las instituciones del país, por lo que han tenido poco alcance $\mathrm{y}$, tal y como indica Rojas (2008), que el país enfrente la investigación científica con estrategias extracurriculares, puede significar el vacío de una pedagogía de investigación en el sistema educativo formal.

\section{MÉTODO}

La presente investigación se considera de tipo descriptiva, por cuanto en ella se delimitaron y definieron las competencias científicas que poseen los estudiantes de grado cuarto y quinto de la institución educativa Gabriel García Márquez, sede B jornada tarde, con un diseño de campo, transeccional contemporáneo univariable. La población estuvo conformada por 102 estudiantes: 52 de cuarto y 50 de quinto de la institución estudiada. Para la recolección de los datos se empleó como instrumento una encuesta con la que se recolectaron los datos aportados por los estudiantes, usando como instrumento una guía de conocimiento la cual está identificado como Anexo A, compuesto por 40 preguntas de selección múltiple, tomadas de las cartillas "Saber" publicadas por el Icfes para grado quinto. La validez se realizó mediante la técnica de validación, por tres expertos en ciencias y la confiabilidad se calculó mediante el Alfa de Cronbach, obteniéndose un valor de 0,75 lo que indica que el instrumento es altamente confiable. Para el análisis de los resultados se utilizó la estadística descriptiva; específicamente la frecuencia absoluta y porcentual, y la mediana como medida de tendencia central por cuanto la variable se midió en un nivel ordinal. Los datos se presentan en tablas y gráficos con su respectiva interpretación. Para ello se calculó un baremo que, por ser una escala convencional de valores utilizada como base para clasificar y ordenar los elementos de un conjunto, permitió evaluar y explicar los resultados obtenidos. (Ver Tabla 1)

Tabla 1. Baremo de interpretación

\begin{tabular}{cc}
\hline Rango & Categorías \\
\hline $0-3,99$ & Muy bajas competencias \\
$4-7,99$ & Bajas competencias \\
$8-11,99$ & Medianas competencias \\
$12-15,99$ & Altas competencias \\
$16-20$ & Muy altas competencias \\
\hline
\end{tabular}

Fuente: Elaboración propia (2020) 


\section{RESULTADOS}

En la Tabla 2 se muestran los resultados de las competencias científicas de los estudiantes con una mediana de 10,21 puntos y un máximo de 15 puntos. Los resultados se ubican en el baremo de interpretación en medianas competencias, lo cual indica que medianamente los estudiantes de los grados 4 to y 5 to son capaces de usar el conocimiento científico para comprender fenómenos que ocurren a su alrededor, interpretar y resolver situaciones científicas.

Tabla 2. Puntaje trasformado de competencias científicas

\begin{tabular}{lcc}
\hline $\mathbf{N}$ & Válidos & $\mathbf{1 0 2}$ \\
\hline Mediana & & 10,21 \\
Mínimo & & 3 \\
Máximo & 25 & 15 \\
& 50 & 8,33 \\
Percentiles & 75 & 10,21 \\
& & 12,50 \\
\hline
\end{tabular}

Fuente: Elaboración propia (2020)

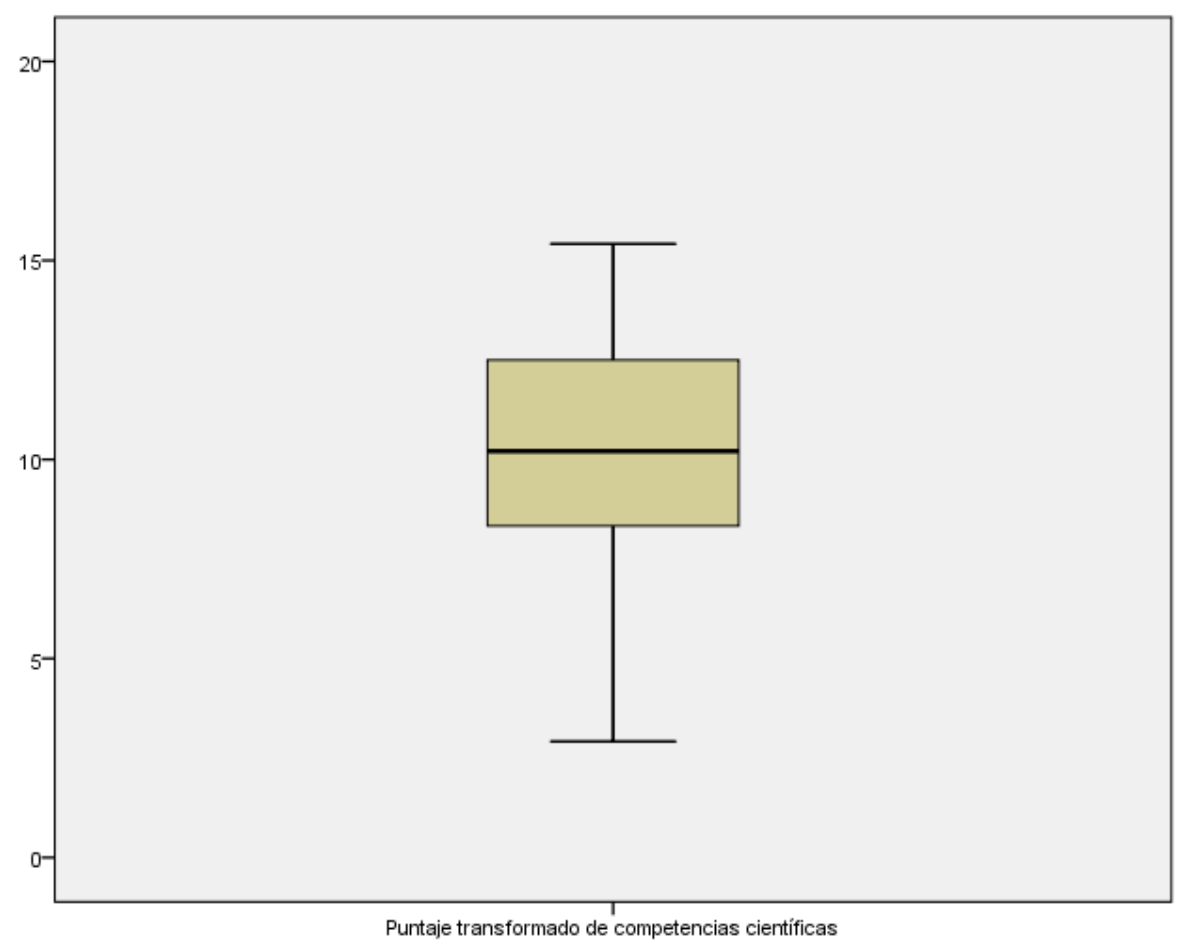

Gráfico 1. Gráfico de caja de bigote de competencias científicas. (Fuente: Elaboración a partir de los datos, 2020) 
De igual manera en la gráfica 1 se observa que la distribución es simétrica y los grupos son homogéneos; es decir, presentan características similares. Sin embargo, el grupo que está por debajo de la mediana es más heterogéneo lo cual implica que presenta características diferentes que el que se encuentra por encima de la mediana.

El valor mínimo de 3 puntos, indica que hubo estudiantes que apenas obtuvieron un puntaje de 3 en la prueba de conocimiento aplicada, lo que está bastante alejado de la mediana $(10,21)$ y mucho más del valor más alto, porque el puntaje máximo son 15 puntos. Eso refleja que los estudiantes que obtuvieron la mayor puntuación, apenas alcanzaron a cubrir el $75 \%$ de los conocimientos científicos referidos a las competencias científicas cognitivas, procedimentales y actitudinales que deberían tener los estudiantes de los grados 4 tos y 5 to.

En cuanto a la distribución de la población en las categorías del evento; es decir, las competencias científicas ubicadas según las respuestas de los estudiantes al cuestionario aplicado, se consiguió, según muestra la Tabla 3, que el 47.1\% del total de jóvenes se ubicó en medianas competencias de acuerdo al baremo de interpretación, y apenas un $32,4 \%$ se ubicó en altas competencias (valor bastante bajo) y un $20,6 \%$ en bajas y muy bajas competencias.

Tabla 3. Categorías de competencias científicas

\begin{tabular}{llcccc}
\hline & Frecuencia & Porcentaje & $\begin{array}{c}\text { Porcentaje } \\
\text { válido }\end{array}$ & $\begin{array}{c}\text { Porcentaje } \\
\text { acumulado }\end{array}$ \\
\hline \multirow{2}{*}{ Muy bajas competencias } & 1 & 1,0 & 1,0 & 1,0 \\
Válidos & Bajas competencias & 20 & 19,6 & 19,6 & 20,6 \\
& Medianas competencias & 48 & 47,1 & 47,1 & 67,6 \\
& Altas competencias & 33 & 32,4 & 32,4 & 100,0 \\
\hline
\end{tabular}

Fuente: Elaboración a partir de los datos

\section{DISCUSIÓN}

Los resultados globales obtenidos de las competencias científicas de los estudiantes de los grados cuarto y quinto de la IE Gabriel García Márquez sede B, muestran que la competencia científica de esos estudiantes se ubica según el baremo de interpretación en medianas competencias, lo cual indica que medianamente los estudiantes de los grados 4 to y 5to, son capaces de usar el conocimiento científico para comprender fenómenos que ocurren a su alrededor e interpretar, además de resolver situaciones científicas.

Esto concuerda con lo expresado por autores como Gallego y otros (2008), quienes afirman que los conocimientos en ciencias de los niños al terminar la primaria son mínimos o nulos, lo cual representa una dificultad en su avance académico, lo que al mismo tiempo afecta 
los resultados recogidos en las pruebas "Saber", donde se reporta que los estudiantes de esos niveles básicos se encuentran en un nivel mínimo en cuanto a las competencias científicas.

A pesar del interés y esfuerzo hecho por el estado colombiano, a través del ente rector en materia educativa, al definir estándares y lineamientos, además de hacer evaluación permanente a través de la prueba "Saber", según los resultados obtenidos, esas expectativas de lograr una educación de calidad, no se alcanzan para las competencias científicas de los estudiantes de la unidad educativa Gabriel García Márquez. De ahí que Serrano (2014) plantea:

La educación tiene que estar atenta al desarrollo de aquellos rasgos de la personalidad humana que son indispensables para el conocimiento y potenciar así en los estudiantes la curiosidad, la búsqueda, la capacidad de sorprenderse, la atención, el interés y el placer por conocer y aprender para elaborar saberes $\mathrm{y}$ compartir posibles soluciones. Cualidades que son esenciales y constituyen, junto a otras, el soporte necesario para la construcción significativa del saber científico. (p.72)

En el caso específico de este estudio, la educación, como principal medio de socialización, debe propiciar la formación integral de las capacidades del ser humano. Debe abrir con mayor énfasis la posibilidad de establecer acciones pedagógicas orientadas a fortalecer las competencias de aprendizaje para el saber hacer, el saber conocer, el saber ser $y$ consecuencialmente el saber convivir de cada persona como bases fundamentales para el desenvolvimiento pleno dentro del contexto colombiano.

\section{CONCLUSIONES}

Los resultados de la competencia científica evaluada muestran que los estudiantes de los grados cuarto y quinto de la institución educativa Gabriel García Márquez del municipio de Soacha, departamento de Cundinamarca poseen conocimientos mínimos o nulos en ciencias, lo que afecta la puesta en práctica de los mismos, ya que, a pesar de mostrar actitudes positivas para esa área del saber, la debilidad en los conocimientos no permite que avancen en la adquisición de nuevas competencias lo que limita la formación de una generación científica de relevo que, contribuya en el futuro, con el desarrollo científico-tecnológico del país.

\section{REFERENCIAS}

Arana, M. (2005). La educación científicotecnológica desde los estudios de la ciencia, tecnología, sociedad e innovación Tabula Rasa. Bogotá. Recuperado de http://www.redalyc.org/articulo.oa?id= 39600315

Banco Mundial. (2014). Apoyo del Banco Mundial a la educación: Un enfoque sistémico para lograr el aprendizaje para todos. Recuperado de https://www.bancomundial.org/es/resu lts/2014/04/28/world-bank-support-toeducation-a-systems-approach-toachieve-learning-for-all

Di Mauro, M., Furman, M. y Bravo, B. (2015). Las habilidades científicas en la escuela primaria: un estudio del nivel de desempeño en niños de 4to año Revista Electrónica de Investigación en Educación en 
Ciencias. Buenos Aires. Recuperado de https://www.researchgate.net/publicati on/290965159_Las_habilidades_cientific as_en_la_escuela_primaria_un_estudio_ del_nivel_de_desempeno_en_ninos_de 4to_ano

Gallego, A., Castro, J. y Rey, J. (2008). El pensamiento científico en los niños y las niñas: algunas consideraciones $e$ implicaciones IIEC. Bogotá. Recuperado de http://cmaps.ucr.ac.cr/rid=1RXS8VTTV1JVFJRL-

3L8/pensamiento\%20cientifico.pdf

Hernández, C. (2005) ¿Qué son las competencias científicas? Foro Educativo Nacional. Bogotá

Instituto Colombiano para la Evaluación de la Educación (Icfes). (2016). Informe nacional, SABER $3^{\circ}, 5^{\circ}$ y $9^{\circ}$ Resultados nacionales 2009 - 2014. Bogotá. Recuperado de https://www.icfes.gov.co/documents /20143/1323329/Resultados\%20nacional es\%20saber\%203\%205\%209\%202009\%202 014.pdf

MEN. (2006) Estándares Básicos de Competencias en Lenguaje, Matemáticas, Ciencias y Ciudadanas. Guía sobre lo que los estudiantes deben saber y saber hacer con lo que aprenden. 2da. edición. MEN, Bogotá. Colombia

MEN. (2004) Estándares Básicos de Competencias en Lenguaje, Matemáticas, Ciencias y Ciudadanas. Guía sobre lo que los estudiantes deben saber y saber hacer con lo que aprenden. 1ra edición. MEN, Bogotá. Colombia

MEN. (1998). Serie Lineamientos Curriculares. Ciencias Naturales y Educación Ambiental. Ministerio de Educación Nacional. Bogotá. Recuperado de https://www.mineducacion.gov.co/1621 /articles-89869_archivo_pdf5.pdf

MEN. (1994) Ley General de Educación. Ley 115 de Febrero 8 de 1994. Recuperado de

http://www.mineducacion.gov.co/1621/arti cles-85906_archivo_pdf.pdf
Pozo, J. y Gómez, M. (2009). Aprender y enseñar ciencia. Del conocimiento cotidiano al conocimiento científico. Madrid: Morata S. L

Ministerio de Educación (2009). Pisa. Programa para la evaluación internacional de los alumnos. OCDE. Informe en español. Madrid, España: Secretaria General Técnica. Recuperado de

https://www.educacionyfp.gob.es/inee/ dam/jcr:bc05a3ce-effe-425b-a79bc92fod43f8d1/pisa-2009-con-escudo.pdf

Rojas, H. (2008). La importancia de las políticas públicas de formación en investigación de niños, niñas y jóvenes en Colombia, para el desarrollo social Revista latinoamericana de Ciencias Sociales, Niñez y Juventud. Manizales. Recuperado de

http://www.redalyc.org/articulo.oa?id= 77360214

Serrano de Moreno, E., Duque de Duque, Y., Madrid de Forero, A. La actividad investigativa en educación media. Representaciones de los profesores sobre las competencias científicas Revista de Pedagogía, 35 (97-98), julio-junio, 2014, pp. 71-91. Universidad Central de Venezuela Caracas, Venezuela

Unesco Office Santiago and Regional Bureau for Education in Latin America and the Caribbean, (2009). Serce. Aportes para la enseñanza de las Ciencia Naturales Pedagogía y Saberes. Chile: Unesdoc. Biblioteca Digital. Recuperado de https://unesdoc.unesco.org/ark:/48223/ pf0000180275

Vallejo, S. (2014). Las competencias científicas en la política educativa colombiana: privilegio de la perspectiva parcial al estudiar su ensamblaje desde los estudios sociales de la ciencia. Bogotá. Colombia 